Corrigendum

\title{
Corrigendum to "Dracohodin Perochlorate Stimulates Fibroblast Proliferation via EGFR Activation and Downstream ERK/CREB and PI3K/Akt/mTOR Pathways In Vitro"
}

\author{
Lin Liu, ${ }^{1}$ Xiaowen Jiang $\mathbb{D}^{1},{ }^{1}$ and Wenhui $Y u \mathbb{D}^{1,2}$ \\ ${ }^{1}$ College of Veterinary Medicine, Northeast Agricultural University, Harbin 150030, China \\ ${ }^{2}$ Key Laboratory of the Provincial Education Department of Heilongjiang for Common Animal Disease Prevention \\ and Treatment, Harbin, China \\ Correspondence should be addressed to Xiaowen Jiang; jiangxiaowen@neau.edu.cn and Wenhui Yu; yuwenhui@neau.edu.cn \\ Received 25 March 2020; Accepted 26 March 2020; Published 28 May 2020 \\ Copyright $\odot 2020$ Lin Liu et al. This is an open access article distributed under the Creative Commons Attribution License, which \\ permits unrestricted use, distribution, and reproduction in any medium, provided the original work is properly cited.
}

In the article titled "Dracohodin Perochlorate Stimulates Fibroblast Proliferation via EGFR Activation and Downstream ERK/CREB and PI3K/Akt/mTOR Pathways In Vitro" [1], there was an error in the Acknowledgments section. The corrected section appears below.

\section{Acknowledgments}

The authors are very grateful to Dr. Guangliang Shi for his help in our experiments. This work was supported by the Natural Science Foundation of China (no. 31572561).

\section{References}

[1] L. Liu, X. Jiang, and W. Yu, "Dracohodin perochlorate stimulates fibroblast proliferation via EGFR activation and downstream ERK/CREB and PI3K/Akt/mTOR pathways in vitro," Evidence-Based Complementary and Alternative Medicine, vol. 2019, Article ID 6027186, 11 pages, 2019. 